\title{
Gestion locale de la diversité variétale du maïs (Zea mays L.) violet par les Tagouana au Centre-Nord de la Côte d'Ivoire
}

\author{
Hugues Annicet N'DA*, Louise AKANVOU et Charles Konan KOUAKOU \\ Centre National de Recherche Agronomique (CNRA) KM 17, Route de Dabou, \\ 01 BP 1740 Abidjan 01, Côte d'Ivoire. \\ *Auteur correspondant ; E-mail: ndahugo@yahoo.fr; Tél: (225) 234724 24; \\ Fax: (225)23 4724 11; Tel.: (225) 08995605
}

\section{RESUME}

La connaissance des pratiques agricoles paysannes liées à la gestion de la diversité des espèces cultivées est nécessaire pour la mise en place d'une stratégie de conservation in situ. L'objectif de la présente étude a été d'appréhender l'état de la diversité variétale du maïs violet et son mode de gestion par les paysans dans la région du Hambol, principale zone de production en Côte d'Ivoire. Vingt quatre (24) villages sélectionnés selon la méthode de recherche participative ont été prospectés. Il ressort des d'enquête que les paysans identifient les variétés exclusivement sur la base de la coloration des grains. Deux (2) noms vernaculaires ont été inventoriés pour désigner trois variétés locales de maïs violet. La culture pure (près de $80 \%$ des enquêtés) constitue le système cultural le plus répandu pour maintenir la pureté variétale. L'étude à souligné aussi que, la faible productivité et la sensibilité à la sécheresse sont à l'origine de l'abandon du maïs violet au détriment du maïs jaune amélioré. La connaissance des contraintes conduisant à un abandon progressif du maïs violet permettra de réguler les introductions variétales dans la région et de préserver ainsi cette variété.

(C) 2013 International Formulae Group. All rights reserved.

Mots clés : Diversité variétale, Variété locale, Hambol, Zea mays, Côte d'Ivoire.

\section{INTRODUCTION}

Le maïs (Zea mays L.) est la première production céréalière dans le monde. Il est une importante ressource alimentaire des régions tropicales et subtropicales. En Côte d'Ivoire, le maïs est la deuxième céréale la plus cultivée après le riz (Oryza spp.). Sa production nationale annuelle est d'environ 654738 tonnes, pour une superficie totale emblavée de 327800 ha (Countrystat, 2013). Une diversité variétale importante est cultivée par les paysans pour diverses raisons. Certaines de ces variétés traditionnelles ont une importance socio-culturelle. C'est par exemple le cas du mais violet, cultivé principalement à Katiola et dans les localités environnantes (Kouakou et al., 2010). Le maïs violet, en plus de sa bonne qualité organoleptique, présente des vertus thérapeutiques (Kouakou et al., 2010). Des études menées par la firme INKANATURAL ont montré que le maïs violet est une source 
naturelle d'anthocyanines, un antioxydant $100 \%$ naturel qui aide à prévenir les maladies cardiovasculaires, à lutter contre le vieillissement cellulaire, à réduire le cholestérol (LDL) et à normaliser la pression artérielle (Inkanatural, 2008). Malgré, cette importance socio-économique et culturelle, très peu de données existent sur le niveau de diversité variétale, la taxonomie locale, le mode de gestion en milieu paysan et les contraintes liées à la culture de cette spéculation en Côte d'Ivoire. Pourtant, de telles informations sont nécessaires pour la mise en place d'une stratégie de conservation in situ. En effet, la gestion des ressources génétiques basée sur les méthodes de conservation ex situ a montré ses limites et de nouvelles stratégies doivent être privilégiées en complément (Sthapit et al., 2010). L'approche utilisée pour compléter les stratégies de conservation ex situ, est la conservation in situ des espèces cultivées par les paysans dans leurs exploitations agricoles (Butare, 2003 ; Dulloo et al., 2010). Subedi et al. (2003) soulignent que les paysans ont de tout temps joué un rôle primordial dans la gestion et la conservation des ressources génétiques. De plus, les travaux effectués à ce jour montrent que la représentation paysanne basée sur les noms des cultivars constitue un point d'entrée important pour l'évaluation de l'agrobiodiversité (Jarvis et al., 2004). La prise en compte de leur connaissance est déterminante et ne doit pas être reléguée au second plan comme le soulignait Brush (1992) en relevant que dans le domaine de la conservation des ressources phytogénétiques, l'implication des populations locales est faible. L'importance de ses savoirs locaux a été confirmée sur plusieurs plantes cultivées comme le sorgho (Sorgum bicolor L. Moench) au Bénin (Missihoun et al., 2012), le fonio millet (Digitaria exilis Stapf, Digitaria iburua Stapf) au Togo (Adoukou-Sagbadja et al.,
2006), les légumes feuilles consommées au Bénin (Dansi et al., 2008). La présente étude a eu pour objectif général d'appréhender l'état de la diversité variétale du maïs violet et son mode de gestion par les paysans dans la région du Hambol en Côte d'Ivoire. Il s'est agi spécifiquement de: (1) faire l'inventaire des variétés locales de maïs violet cultivées, (2) décrire le procédé d'identification local du maïs violet, (3) déterminer les stratégies mises en œuvre par les agriculteurs pour les conserver et (4) inventorier les facteurs qui déterminent le maintien ou la disparition de cette variété locale.

\section{MATERIEL ET METHODES \\ Site d'étude}

Le département de Katiola est situé dans la région du Hambol, au Centre-Nord de la Côte d'Ivoire (Figure 1). Il est situé entre $8^{\circ} 10^{\prime}$ de latitude Nord et $5^{\circ} 4^{\prime}$ de longitude Ouest et occupe une superficie de $9420 \mathrm{~km}^{2}$ (Kouakou et al., 2010). La pluviométrie annuelle oscille entre $1100 \mathrm{~mm}$ et $1200 \mathrm{~mm}$ (Ouattara, 1998). Les températures moyennes enregistrées dans cette zone varient entre 26,45 et 33, $67^{\circ} \mathrm{C}$ (Dembélé, 2008). Les sols sont de types ferrallitiques dominés par des sols à texture argilo-sableuse et gravillonnaire (Soro et al., 2011). La végétation est celle des savanes pré-forestières. Elle appartient pour la majorité des cas à celle du secteur subsoudanais du domaine soudanais. La commune de Katiola a été choisie parce qu'elle est une zone de production du maïs violet en Côte d'Ivoire.

\section{Méthodologie de collecte des données}

La présente étude s'inscrit dans la continuité d'autres enquêtes menées dans la même zone par Kouakou et al. (2010). Sur la base de ces enquêtes préliminaires, 3 localités ont été retenues pour l'étude. Il s'agit de Katiola, Niakaramadougou et Fronan. Les 
enquêtes ont été conduites suivant un guide questionnaire d'après Dansi et al. (2011). Ce guide a abordé les contraintes liées à la culture du maïs «violet» dans la zone d'étude, la diversité variétale cultivée, les pratiques culturales, la taxonomie locale etc. En fonction de la taille des localités, 3 à 12 villages ont été retenus soit au total 24 villages enquêtés. Les données ont été collectées à travers des entretiens en groupes et individuels (structurés et non structurés), complétées par des observations directes sur le terrain. Dans l'ensemble 100 paysans ont été enquêtés. La liste des villages enquêtés et leur localisation administrative (régions et département) sont consignées dans le Tableau 1.

\section{Analyse des données}

Les données collectées ont été analysées par la statistique descriptive à l'aide du logiciel SPSS (version 17). Les résultats sont présentés sous la forme de graphiques et de tableaux réalisés grâce à Excell (version 2007). Des Analyses Factorielles des Correspondances (AFC) ont également été réalisées à l'aide du logiciel Statistica 7 (version 7.1).

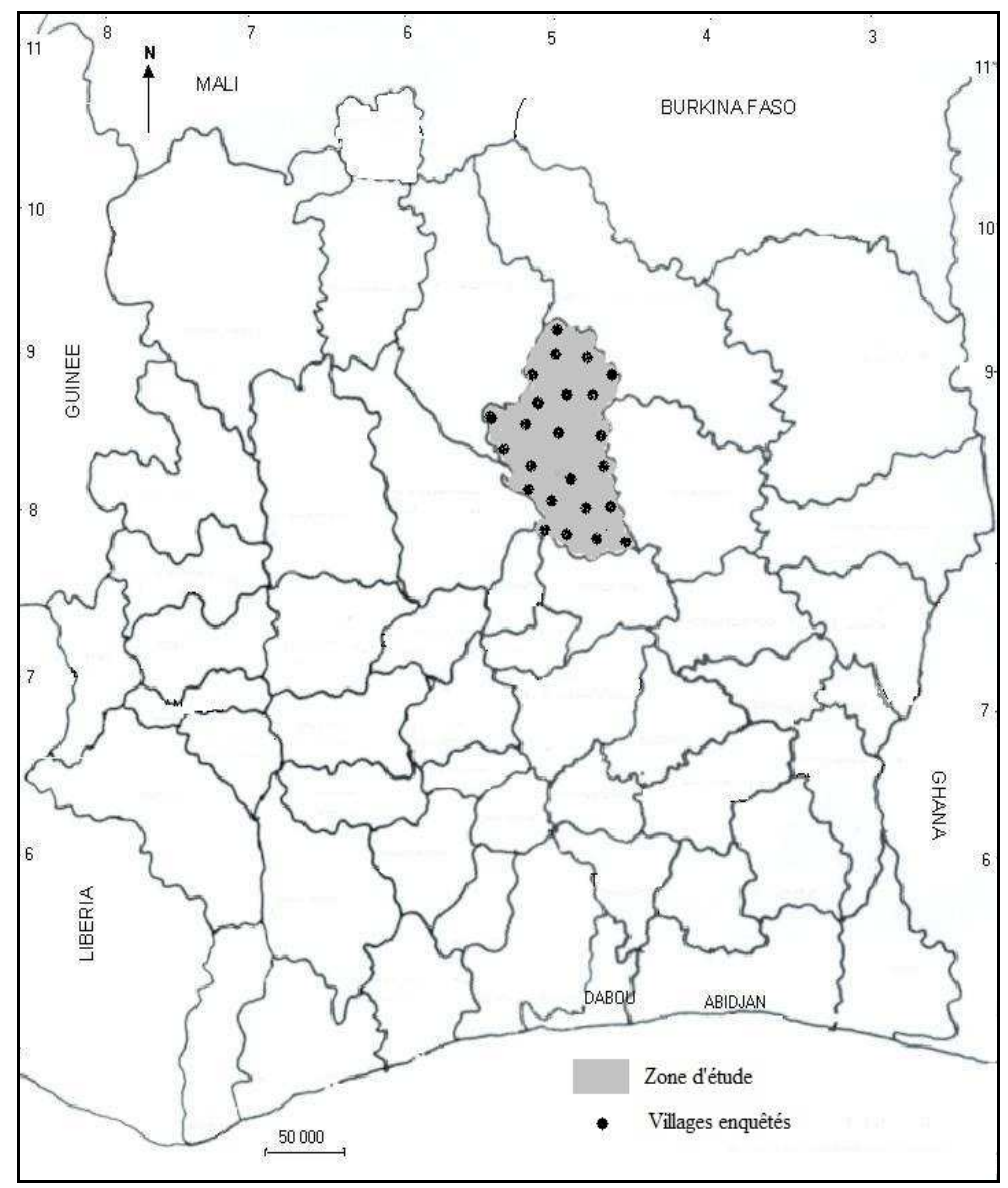

Figure 1 : Carte de la Côte d'Ivoire présentant les villages prospectés. 
Tableau 1 : Localisation administrative et groupes ethniques rencontrés dans les villages visités.

\begin{tabular}{lcccc}
\hline $\mathbf{N}^{\circ}$ & Villages & Départements & Région & Groupe ethnique \\
\hline 1 & Longo & Niakaramadougou & Hambol & Tagouana \\
2 & Kanawôlo & Niakaramadougou & Hambol & Tagouana \\
3 & Sinkaha & Niakaramadougou & Hambol & Tagouana \\
4 & Latokaha & Niakaramadougou & Hambol & Tagouana \\
5 & Pekaha & Niakaramadougou & Hambol & Tagouana \\
6 & Kafiné & Niakaramadougou & Hambol & Tagouana \\
7 & Timorokaha & Niakaramadougou & Hambol & Tagouana \\
8 & Angolokaha & Niakaramadougou & Hambol & Tagouana \\
9 & Pétionnara & Niakaramadougou & Hambol & Tagouana \\
10 & Oureguekaha & Niakaramadougou & Hambol & Tagouana \\
11 & Ounandiekaha & Fronan & Hambol & Tagouana \\
12 & Darakokaha & Fronan & Hambol & Tagouana \\
13 & Perabohinkaha & Katiola & Hambol & Tagouana \\
14 & Logbonou & Katiola & Hambol & Tagouana \\
15 & Nahobankaha & Katiola & Hambol & Tagouana \\
16 & Kouamékaha & Katiola & Hambol & Tagouana \\
17 & Touro & Katiola & Hambol & Tagouana \\
18 & Kafigué & Katiola & Hambol & Tagouana \\
19 & Ourougbankaha & Katiola & Hambol & Tagouana \\
20 & Kabolo & Katiola & Hambol & Tagouana \\
21 & Kationon & Katiola & Hambol & Tagouana \\
22 & Tiengala & Fronan & Hambol & Tagouana \\
23 & Kpefelé & Katiola & Hambol & Tagouana \\
24 & Koffissiokaha & Katiola & Hambol & Tagouana \\
\hline
\end{tabular}

\section{RESULTATS}

Diversité variétale et nomenclature locale

Trois (3) variétés locales de maïs violet ont été observées dans les différentes localités enquêtées. Il s'agit des maïs à grain violet foncé, des maïs à grain violet clair et des maïs à grain violet clair qui tire sur le rouge (Figure 2).

La dénomination en langue vernaculaire des différentes variétés est consignée dans le Tableau 2. Le maïs violet foncé est appelé Nandéwô tandis que Nandé n'kon est utilisé pour désigner le maïs violet clair et le maïs violet clair qui tire sur le rouge.

\section{Distribution et importance des variétés}

La Figure 3 montre la fréquence d'apparition de chaque type variétal. Sur les 100 agriculteurs enquêtés, la variété Nandéwô est cultivée par $60 \%$ (60/100), contre $40 \%$ (40/100) pour la variété Nandé n'kon.

\section{Origine des semences}

La proportion relative des modes d'acquisition des semences par les agriculteurs des localités visitées est présentée dans le Tableau 3. Sur l'ensemble des agriculteurs enquêtés, 47,2\% ont acquis leur semence par achat sur le marché, 45,3\% par héritage et $7,5 \%$ par don. Le mode d'acquisition des semences varie aussi selon l'âge. Parmi les paysans interrogés, les personnes âgées (50 ans et plus), avec une fréquence de 52,8\%, affirment avoir obtenu leurs semences par héritage. Ce qui n'est pas le cas pour les jeunes (18-25 ans) et les adultes (26-49 ans) dont le marché constitue la principale source d'approvisionnement (Tableau 3). 
Par ailleurs, une relation statistiquement significative (Khi-deux de pearson $\mathrm{p}=0,012<0,05)$ mais faible ( $\mathrm{V}$ de cramer $=0,348)$ a été mis en évidence entre le mode d'acquisition et les localités prospectées (Tableau 4). D'une manière générale, l'acquisition des semences par achat est le mode le plus dominant à Niakaramadougou $(73,7 \%)$ alors qu'à Katiola $(65 \%)$ et à Fronan $(57,1 \%)$, c'est le mode d'acquisition par héritage qui est le plus pratiqué. Le don est quasi inexistant à Katiola et faiblement pratiqué à Niakaramadougou $(10,5 \%)$ et à Fronan $(14,3 \%)$.

Principales raisons de l'abandon de la culture du maïs violet dans la zone d'étude

Les principales contraintes évoquées par les agriculteurs au cours des enquêtes sont présentées à la Figure 4. La faible productivité (29\%) et la sensibilité à la sécheresse (29\%) constituent les contraintes majeures à la production de cette variété. Le manque de débouchés, la destruction des champs par les bœufs, la sensibilité aux insectes, l'introduction du maïs jaune, la baisse de la fertilité du sol et la culture d'anacardier sont les autres contraintes les plus fréquemment citées.

D'autre part une analyse factorielle des correspondances multiples à été effectuée pour montrer la relation qui existe entre les localités et les contraintes enregistrées (Figure 5). A Niakaramadougou, le manque de débouchés et les dégâts occasionnés par les bœufs sont les principales raisons de l'abandon de la culture du maïs violet au profit d'autres variétés plus rentables économiquement comme le maïs jaune. A
Katiola par contre, la baisse de la fertilité ainsi que la grande sensibilité à la sécheresse sont les problèmes les plus mentionnés par les paysans. A Fronan, les agriculteurs trouvent que le maïs violet est moins productif que la variété jaune introduite dans la zone par l'Agence Nationale d'Appui au Développement rural (ANADER).

La Figure 6 présente les raisons pour lesquelles certains paysans maintiennent encore la culture du maïs violet. Les deux premières raisons sont la précocité et la qualité du tôh (semoule de maïs). En effet, pour $100 \%$ des enquêtés le maïs violet est précoce et présente un très bon goût. Environ $59 \%$ des agriculteurs affirment aimer cette variété à cause du bon tchapalo (Bière traditionnelle à base de maïs, mil ou sorgho) qu'on en tire et aussi à cause de sa bonne valeur marchande comparée aux autres variétés cultivées dans la région. Pour d'autres, c'est par habitude alimentaire ou par préjugé (le maïs jaune ou blanc est considéré comme un totem dans la famille) qu'ils cultivent encore cette variété.

\section{Pratiques culturales et maintien de la pureté variétale}

Les pratiques culturales de maintien de la pureté variétale du maïs violet par les agriculteurs et leurs fréquences sont données dans la Figure 7. Environ $80 \%$ des paysans cultivent le maïs violet en l'isolant des autres variétés qu'ils cultivent, contre $20 \%$ qui pratique la culture associée. Chez certains paysans pratiquant la culture mono-variétale, des épis bigarrés ont été observés à une fréquence de $25 \%$.

Tableau 2 : Désignations locales du maïs violet et leurs significations en Tagouana.

\begin{tabular}{ccccc}
\hline $\mathbf{N}^{\circ}$ & Désignation locale & $\begin{array}{c}\text { Autres } \\
\text { désignations }\end{array}$ & Groupe ethnique & Signification \\
\hline 1 & Nandéwồ & - & Tagouana & Maïs noir (violet foncé) \\
2 & Nandé n'kon & - & Tagouana & Maïs clair (violet clair) \\
\hline
\end{tabular}


Tableau 3 : Relation entre les modes d'acquisition et les tranches d'âge.

\begin{tabular}{|c|c|c|c|c|c|c|}
\hline & & & \multicolumn{3}{|c|}{ Tranches d'âge } & \multirow{2}{*}{ Total } \\
\hline & & & $\begin{array}{l}\text { Personnes âgées } \\
\text { (50 ans et plus) }\end{array}$ & $\begin{array}{c}\text { Adultes } \\
(26-49 \text { ans })\end{array}$ & $\begin{array}{c}\text { Jeunes } \\
\text { (18-25 ans) }\end{array}$ & \\
\hline \multirow{3}{*}{$\begin{array}{l}\text { Modes } \\
\text { d'acquisition }\end{array}$} & Achat sur le marché & $\%$ within Trage & $41,7 \%$ & $60,0 \%$ & $57,1 \%$ & $47,2 \%$ \\
\hline & Héritage & $\%$ within Trage & $52,8 \%$ & $40,0 \%$ & $14,3 \%$ & $45,3 \%$ \\
\hline & Don & $\%$ within Trage & $5,6 \%$ & & $28,6 \%$ & $7,5 \%$ \\
\hline Total & & $\%$ of Total & $67,9 \%$ & $18,9 \%$ & $13,2 \%$ & $100,0 \%$ \\
\hline
\end{tabular}

Tableau 4 : Relation entre les modes d'acquisition et les localités d'étude.

\begin{tabular}{|c|c|c|c|c|c|c|}
\hline & & & \multicolumn{3}{|c|}{ Localités } & \multirow[b]{2}{*}{ Total } \\
\hline & & & Niakara & Fronan & Katiola & \\
\hline \multirow{3}{*}{$\begin{array}{l}\text { Modes } \\
\text { d'acquisition }\end{array}$} & Achat sur le marché & $\%$ within Local & $73,7 \%$ & $28,6 \%$ & $35,0 \%$ & $47,2 \%$ \\
\hline & Héritage & $\%$ within Local & $15,8 \%$ & $57,1 \%$ & $65,0 \%$ & $45,3 \%$ \\
\hline & Don & $\%$ within Local & $10,5 \%$ & $14,3 \%$ & & $7,5 \%$ \\
\hline Total & & $\%$ within Local & $100,0 \%$ & $100,0 \%$ & $100,0 \%$ & $100,0 \%$ \\
\hline
\end{tabular}

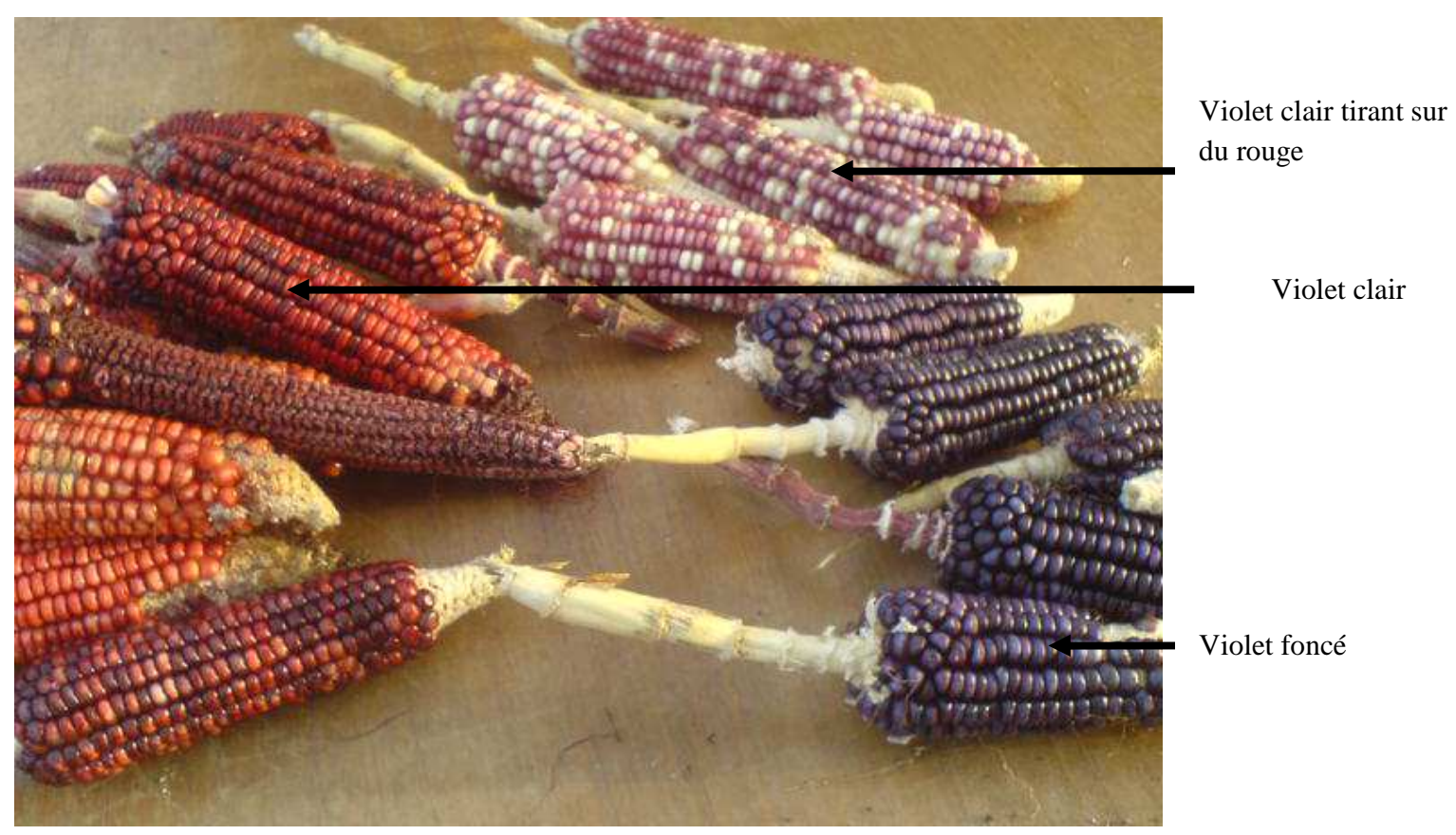

Figure 2 : Différentes variétés de maïs «violet» sur la base de la couleur des grains. 


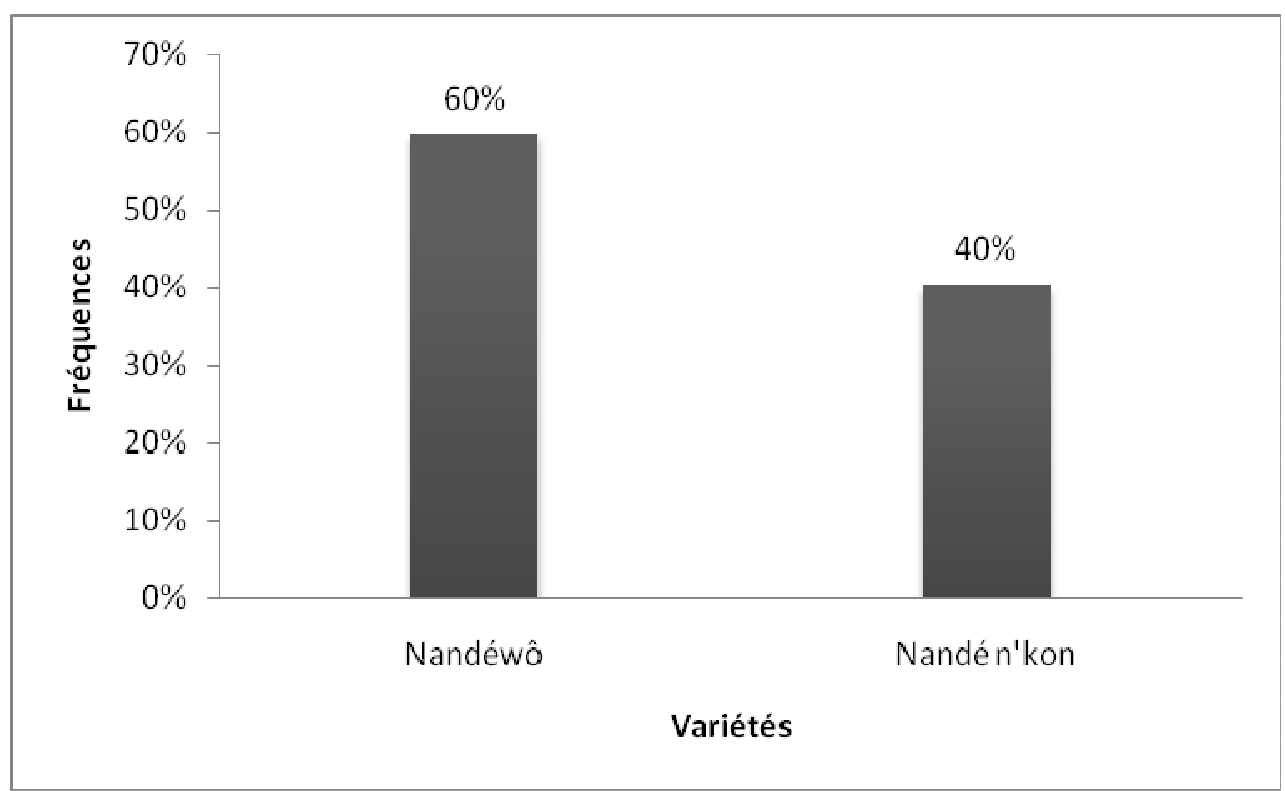

Figure 3 : Fréquence des différentes variétés de maïs «violet» dans la zone d'étude.

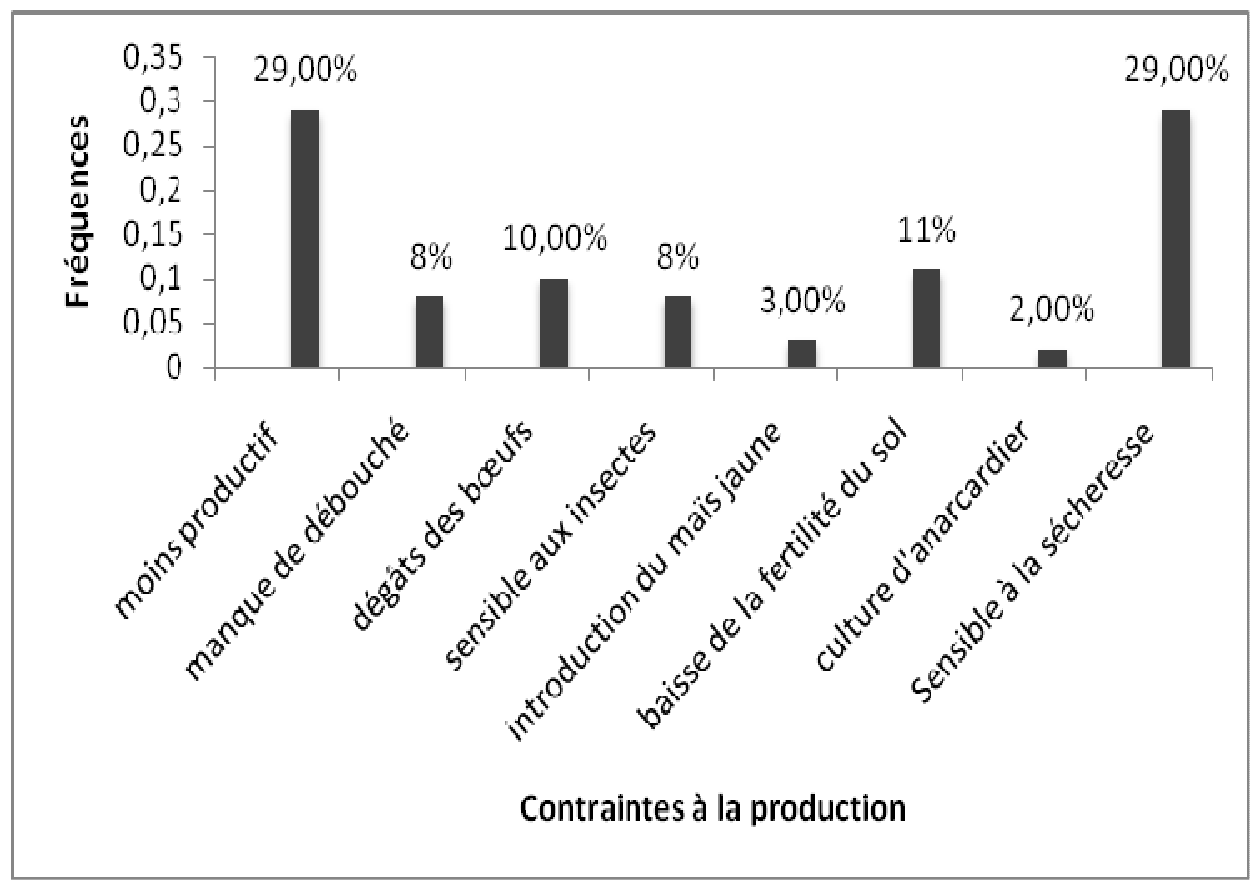

Figure 4: Principales contraintes liées à la production du maïs violet en Côte d'Ivoire. 


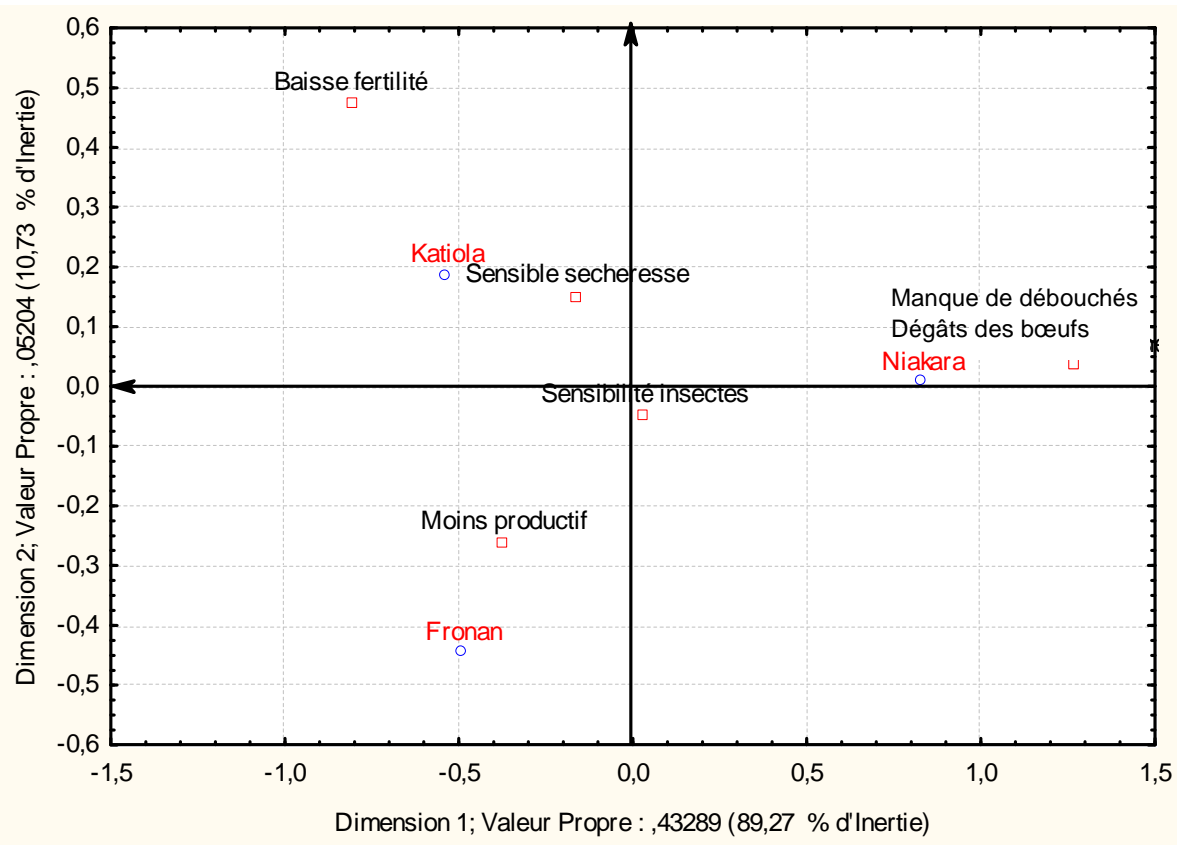

Figure 5: Relations entre les principales contraintes évoquées par les paysans et les localités enquêtées en Côte d'Ivoire.

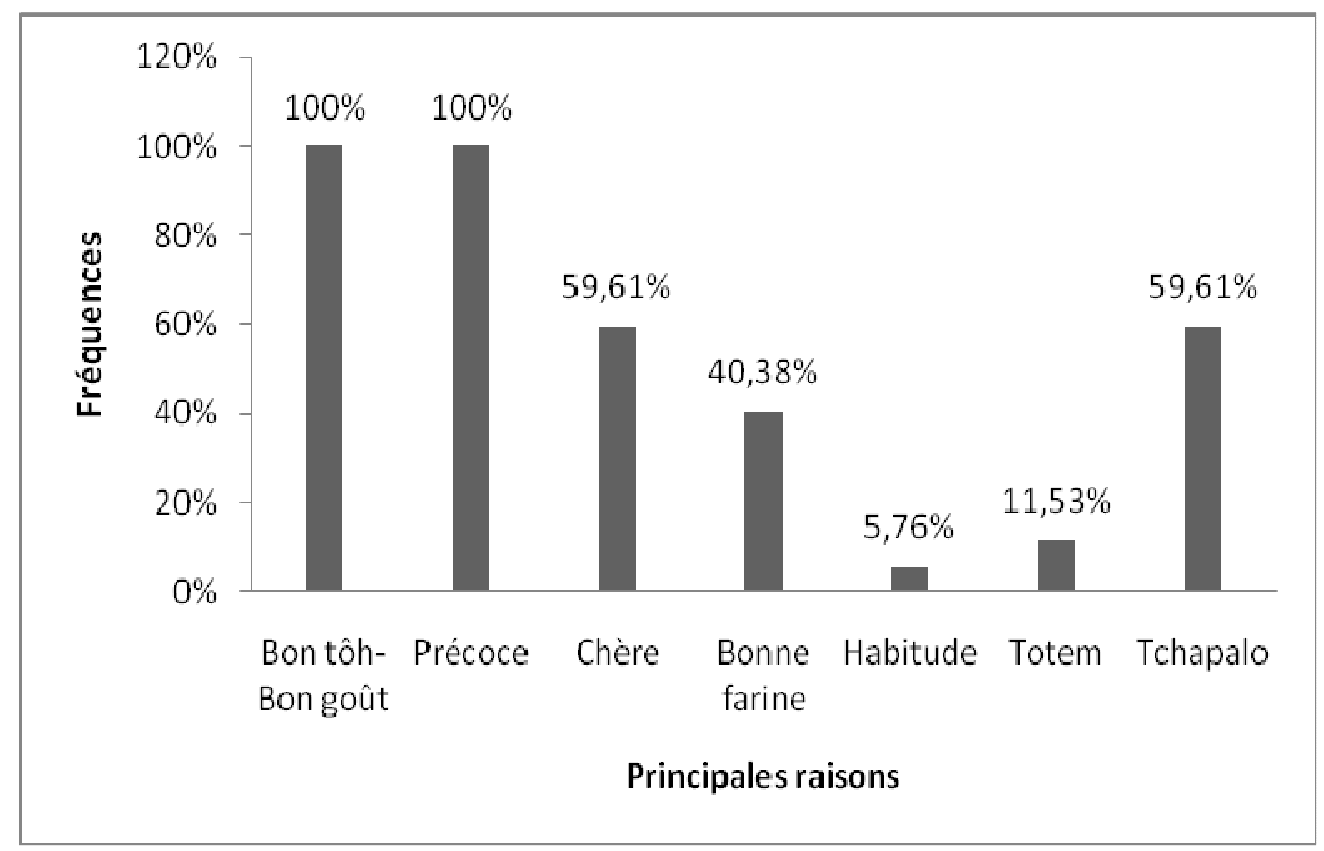

Figure 6 : Principales raisons de la culture du maïs violet par les paysans dans les zones enquêtées. 


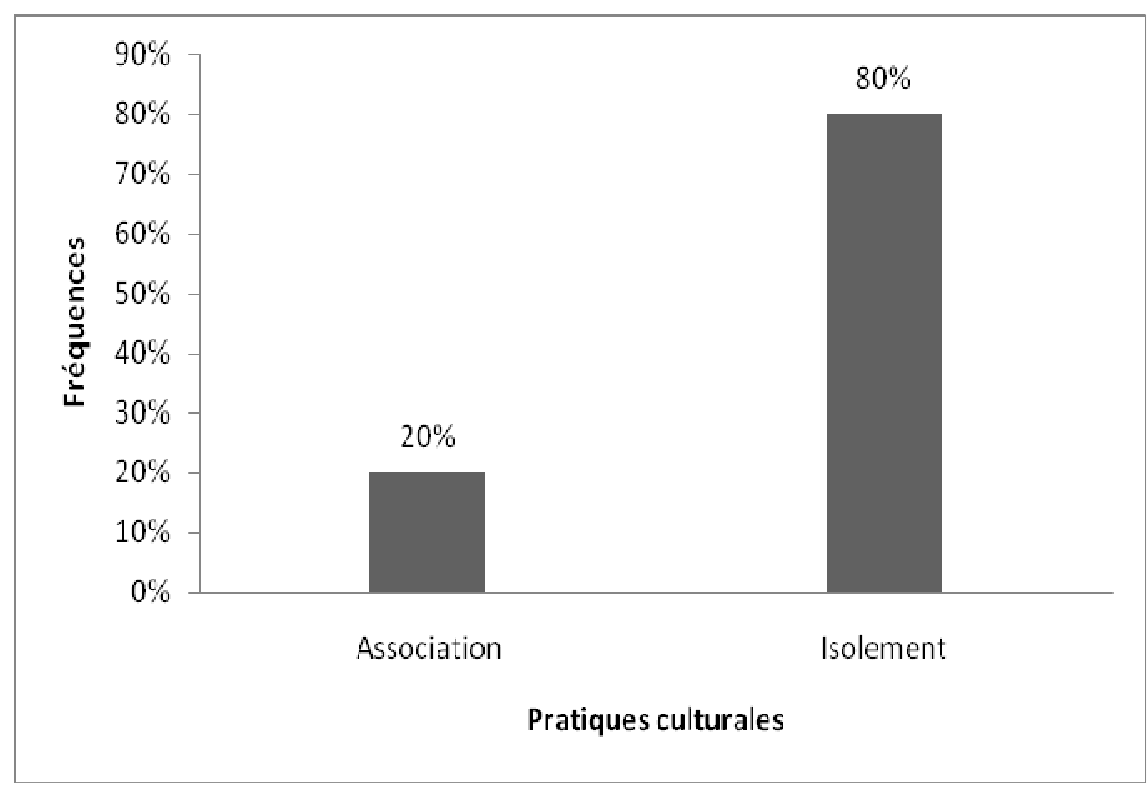

Figure 7 : Pratiques culturales et maintien de la pureté variétale du maïs violet selon les paysans interrogés.

\section{DISCUSSION}

Chez les Tagouana du Centre-Nord de la Côte d'Ivoire, le nom générique du maïs est Nandé. Sur la base de l'intensité de la coloration des grains trois variétés locales de maïs violet ont été observés dans la présente étude. Ces derniers sont désignés par deux noms vernaculaires (Nandéwô et Nandé n'kon). L'importance de la couleur dans la description et la dénomination des variétés locales a été mise en évidence chez plusieurs espèces végétales. Missihoun et al. (2012), ont montré qu'au Nord-Ouest du Bénin, la couleur des grains et la durée du cycle constituent les critères les plus importants utilisés par les producteurs pour identifier, nommer et classer leurs variétés de Sorgho. De même, Tostain et al. (2002) dans une étude réalisée au Sud du Bénin, ont montré que la couleur du parenchyme amylacé permettait aux paysans de distinguer trois variétés d'igname Gban. Cela pourrait s'expliquer par le fait que la couleur est un caractère facilement perceptible par les paysans. En dehors de la coloration des grains, aucun autre critère n'a été évoqué par les paysans pour désigner leurs variétés. Ces résultats corroborent ceux de Kouakou et al. (2010). Par ailleurs, les enquêtes ont révélées que la variété Nandéwô avec un pourcentage de $60 \%$ est la plus cultivée par les ménages. Mais, il n'est pas rare de rencontrer les différents types dans un même ménage. L'importance relative de la variété Nandéwô serait liée à ses vertus thérapeutiques. En effet, selon les paysans interrogés plus le maïs est noir plus il ralentirait le vieillissement. Ces résultats sont similaires à ceux de Kouakou et al. (2010). Toutefois, pour certains paysans il s'agirait des mêmes variétés. La coloration violet clair serait liée à un phénomène lunaire. Pour ces derniers, lorsque le semis survient au lendemain de la pleine lune, il apparait dans la descendance des individus clairs. Ce qui laisse présager, sous réserve d'une évaluation plus approfondie, que Nandé n'kon (violet clair) est une variante de Nandéwô (Violet foncé) qui par sélection humaine est devenue une variété à part entière. Cette pratique presque semblable à celle observée en Amazonie avec le manioc (McKey et al., 2001) contribue à l'augmentation de la diversité génétique de la variété. Les semences des paysans enquêtés 
proviennent de différentes sources. Mais, l'achat sur le marché représente la source la plus importante (environ 47,2\%). Ces résultats sont différents de ceux de Kouakou et al. (2010) qui ont travaillé dans la même région et de ceux de Missihoun et al. (2012) sur le sorgho au Bénin. Selon les premiers auteurs, $76 \%$ des paysans ont obtenu leurs semences par héritage et seulement $6 \%$ les ont obtenues par achat sur le marché alors que pour les deuxièmes $83,15 \%$ des producteurs ont acquis leurs semences par héritage contre seulement $8,99 \%$ (étrangers ou de retour d'une aventure d'un pays voisin) ont acquis leurs semences par achat. La différence entre les résultats obtenus dans la présente étude et ceux de ces auteurs pourrait être liée à la taille de l'échantillon. D'autre part, la faible productivité et la sensibilité à la sécheresse du maïs violet semble être à l'origine de son abandon par plusieurs paysans au profit des variétés à grain jaune améliorées introduites dans la région par la recherche. Une étude récente, conduite par Baco et al. (2011) sur les ménages producteurs de maïs au Bénin, a montré que les variétés locales sont moins rentables que les variétés améliorées, du point de vue des utilisateurs. Ce résultat, similaire au nôtre, est d'autant plus préoccupant qu'on assiste aujourd'hui à la disparition du maïs violet de ces régions. Des efforts doivent être déployés pour préserver cette variété locale, notamment par l'amélioration de sa productivité. Par ailleurs, Environ $80 \%$ des paysans cultivent le maïs violet en l'isolant des autres variétés qu'ils cultivent. Kouakou et al. (2010) ont montré des résultats similaires. Selon ces auteurs, 90\% des producteurs interrogés affirment cultiver le maïs violet en culture mono-variétale pure. Néanmoins, l'observation d'épis bigarrés dans le cas de la présente étude témoigne d'une hybridation entre les variétés. Cette situation pourrait être liée à la proximité des champs. En effet, pour maintenir la pureté variétale chez une espèce allogame comme le maïs, la distance d'isolement requise est comprise entre 200 et $400 \mathrm{~m}$ (Bono, 1981). Or, en milieu paysan les contraintes de terre et le manque de formation des agriculteurs font que cette distance n'est pas respectée.

\section{Conclusion}

La présente étude à montré que trois variétés de maïs violet sont cultivés dans la région du Hambol. La dénomination de ces variétés par les Tagouana est basée exclusivement sur l'intensité de la coloration des grains. La faible productivité et la sensibilité à la sécheresse entraînent aujourd'hui un abandon progressif de la culture du maïs violet au profit des variétés de maïs à grains jaunes introduites dans la région par la recherche. Des actions visant la préservation de cette ressource phytogénétique importante doivent être envisagées. Des efforts doivent également être menés pour préserver le mode de maintien de la pureté du maïs violet, par la formation des agriculteurs à la production de semences.

\section{REMERCIEMENTS}

Nous tenons à remercier très sincèrement le Fonds Interprofessionnel pour la Recherche et le Conseil Agricoles (FIRCA), pour avoir financé ce travail à travers le projet FIRCA-WAAPP 'Reconstitution des ressources génétiques du maïs',

\section{REFERENCES}

Adoukonou-Sagbadja H, Dansi A, Vodouè R, Akpagana K. 2006. Indigenous knowledge and traditional conservation of Fonio millet (Digitaria exilis Stapf, Digitaria iburua Stapf) in Togo. Biodivers. Conserv., 15: 2379-2395.

Baco MN, Abdoulaye T, Sanogo D, Langyintuo A. 2010. Caractérisation des ménages producteurs de maïs en zone de savane sèche au Bénin. Rapport pays- Enquête-ménage - Benin.

Brush SB. 1992. Ethnoecology, biodiversity and modernization. In Andean potato agriculture. J. Ethnobiol., 12 : 161-185. 
Bono M. 1981. Multiplication des Semences Vivrières Tropicales. Presses Universitaires de France: Paris.

Butare I. 2003. La biodiversité en Afrique de l'Ouest: Leçons apprises et perspectives. La biodiversité agricole en Afrique de l'ouest, situation actuelle, expériences et perspectives. FAO, Bamako, Mali.

Countrystat. 2013. http//: www.countrystat. org/civ/cont/pxwebquery/ma/107spd080/ fr.

Dansi A, Adjatin A, Adoukonou-Sagbadja H and Akpagana K. 2008. Production and traditional seed conservation of leafy vegetables in Benin rural areas. Bulletin de la Recherche Agronomique du Bénin, 59: 59-69.

Dansi A, Dantche H. 2011. Increased farmers and breeders access to yam diversity in Togo. Rapport du projet.

Dembélé DD. 2008. Effets des urines humaines hygiénisées sur la productivité du riz irrigué, Oryza Sativa variété « Bouaké 189 », à Katiola (Centre nord de la Côte d'Ivoire). Mémoire de DESS, Université Abdou Moumouni, Niger, p 56.

Dulloo ME, Hunter D, Borelli T. 2010. Ex Situ and In Situ Conservation of Agricultural Biodiversity: Major Advances and Research Needs. Not. Bot. Hort. Agrobot. Cluj., 38(2) : 123-135.

Inkanatural. 2008. Extrait maïs violet, 100 gélules. http://www.inkanatural.com.

Jarvis DI, Zoes V, Nares D, Hodgkin T. 2004. On-farm management of crop genetic diversity and the Convention on Biological Diversity programme of work on agricultural biodiversity. Plant Genetic Resour. Newsl., 138: 5-17.
Kouakou CK, Akanvou L, Konan YA, Mahyao A. 2010. Stratégies paysannes de maintien et de gestion de la biodiversité du maïs (Zea mays L.) dans le département de Katiola, Côte d'Ivoire. $J$. Appl. Biosci., 33: 2100 - 2109.

McKeye D, Emperaie L, Elias M, Pinton F, Robert T, Desmoulière S, Rival L. 2001. Gestion locales et dynamiques régionales de la diversité variétale du manioc en Amozonie. Genet. Sel. Evol., 33 : 465-490.

Missihoun AA, Agbangla C, AdoukonouSagbadja H, Ahanhanzo C, Vodouhè R. 2012. Gestion traditionnelle et statut des ressources génétiques du sorgho (Sorghum bicolor L. Moench) au NordOuest du Bénin. Int. J. Biol. Chem. Sci., 6: $1003-1018$.

Soro D, S Bakayoko, Dao D, TrabT i, Angui P, Girardin O. 2011. Diagnostic de fertilité du sol au Centre-Nord de la Côte d'Ivoire. Agronomie Africaine, 23(3): 205-215.

Sthapit B, Padulosi S, Mal M. 2010. Role of on-farm/In situ conservation and underutilized crops in the wake of climate change. Ind. Jour. Plant Genet. Resour., 23(2) : 145-156.

Subedi A, Chaudary P. 2003. Who maintains crop genetic diversity and how: Implications for on-farm conservation and utilization. Culture Agric., 25(2): 41-50.

Tostain S, Baco MN, Okry FK, Mongbo RL, Agbangla C, Dainou O. 2002. La gestion dynamique des variétés d'igname dans le système traditionnel de production au Bénin. Annales des Sciences Agronomique du Bénin, 3(2): 73-93. 